\title{
Erratum to "Multilevel B-Spline Repulsive Energy in Nanomodeling of Graphenes" [Journal of Surface Engineered Materials and Advanced Technology Vol. 4 No. 2 (April 2014) 75-86]
}

\author{
Maharavo Randrianarivony \\ Virtual Material Design, Fraunhofer Institute for Algorithms and Scientific Computing SCAI, Schloss \\ Birlinghoven, Sankt Augustin, Germany \\ Email: maharavo.randrianarivony@scai.fraunhofer.de
}

Received 6 January 2014; revised 5 February 2014; accepted 4 March 2014

Copyright (C) 2015 by author and Scientific Research Publishing Inc.

This work is licensed under the Creative Commons Attribution International License (CC BY).

http://creativecommons.org/licenses/by/4.0/

(c) ()

The original online version of this article (Randrianarivony, M. (2014) Multilevel B-Spline Repulsive Energy in Nanomodeling of Graphenes. Journal of Surface Engineered Materials and Advanced Technology, Vol. 4 No. 2, 75-86. doi: 10.4236/jsemat.2014.42011) did not contain any acknowledgment. The author wishes to add the following acknowledgment:

\section{Acknowledgements}

This work was partially supported by Eurostars Project E!6935 funded by German Federal Ministry of Education and Research.

In addition, please remove Figure 5 because it has little relevance with the proposed method. Discard also the corresponding description on page 84: "As a next test, we consider the complex band structures for using the DFT and SE computations whose results are respectively displayed in Figure 5(a), Figure 5(b) for the graphene with chirality $(1,0)$. The plots depict band lines which are not shown as continuous curves but as sets of sampling points. The points which are purely real and explicitly complex are depicted in red and green respectively”. 